\title{
ESTILOS DE APRENDIZAJE VAK Y HONEY-ALONSO DE LOS \\ ESTUDIANTES DE QUÍMICO FARMACÉUTICO BIÓLOGO DE LA UNIVERSIDAD AUTÓNOMA DE CAMPECHE, GENERACIÓN 2017
}

\section{VAK AND HONEY-ALONSO \\ LEARNING STYLES OF BIOLOGIST \\ PHARMACEUTICAL CHEMIST STUDENTS FROM AUTONOMOUS UNIVERSITY OF CAMPECHE, 2017 GENERATION}

\author{
Marvel del Carmen Valencia Gutiérrez ${ }^{1}$ \\ Pedro Francisco Ávila Peraza² \\ Magnolia del Rosario López Méndez \\ María de Jesús García Ramírez ${ }^{4}$ \\ Universidad Autónoma de Campeche ${ }^{1}$ \\ Instituto Tecnológico de Campeche ${ }^{2}$ \\ San Francisco de Campeche, México
}




\section{RESUMEN}

En esta investigación se realizó un estudio y se usó el modelo de Honey y Alonso también llamado CHAEA y el Modelo de la Programación Neurolingüística de Bandler y Grinder del canal Visual, Auditivo, Kinestésico, también llamado VAK a los 92 estudiantes que ingresaron en la generación 2017 en el Programa Educativo de Químico Farmacéutico Biólogo de la Facultad de Ciencias Químico Biológicas de la Universidad Autónoma de Campeche. Los resultados muestran que el estilo predominante en los tres grupos de la generación de ingreso 2017 es el Reflexivo y Auditivo de acuerdo a las dos clasificaciones de estilos que se les aplicó a los estudiantes. Estos resultados son informados a los docentes del Programa Educativo para considerarlo en el diseño de sus estrategias de enseñanza, fomentando así el uso de los estilos de los estudiantes en el proceso de enseñanza y aprendizaje en el aula, lo que fortalecerá el nivel de aprovechamiento escolar en el Nivel Superior.

PALABRAS CLAVE: Estilos, Aprendizaje, Honey Alonso, VAK

\section{ABSTRACT:}

This research was conducted by the model of Honey and Alonso also called CHAEA and also the Model of the Neuro-linguistic Programming

1 mcvalenc@uacam.mx Universidad Autónoma de Campeche ${ }^{1}$ Tecnológico de Campeche ${ }^{2}$ San Francisco de Campeche, México 9817504644 ORCID: 0000-0002-36710296

$2 \quad$ Universidad Autónoma de Campeche 1 Tecnológico de Campeche ${ }^{2}$ San Francisco de Campeche, México 9811050162 pedroavila18@hotmail.com ORCID: 00000002-8209-2417

$3 \quad$ Universidad Autónoma de Campeche ${ }^{1}$ Tecnológico de Campeche ${ }^{2}$ San Francisco de Campeche, México. marlopez@uacam.mx 9811108014 ORCID: 0000-00027919-894X

$4 \quad$ mjgarcia@uacam.mx 9821091426. Universidad Autónoma de Campeche ${ }^{1}$ Tecnológico de Campeche ${ }^{2}$ San Francisco de Campeche ORCID: 0000-0002-2707-8081 of Bandler and Grinder of the channel Visual, Auditory, Kinesthetic, also called VAK. The sample was made up of 92 students who entered in the 2017 generation in the Instructional Program of Pharmaceutical Chemical Biologist at the Faculty of Chemical and Biological Sciences at the Autonomous University of Campeche. The results show that the predominant style in the three groups of income generation 2017 is the reflective and auditory styles according to the two classifications of styles applied to them to students. These results were reported to the teachers of the educational program for consideration in the design of their teaching strategies, promoting the use of the styles of students in the process of teaching and learning in the classroom, which will strengthen the level of achievement in the upper level.

KEYWORDS: Styles, Learning, Honey Alonso, VAK.

\section{INTRODUCCIÓN}

La educación superior debe reconocer y potenciar al estudiante como centro y motor de su propio proceso formativo, lo cual requiere sumar esfuerzos no sólo por parte de las instituciones encargadas de la formación del educando que llega a sus aulas, sino también por parte del profesorado que, para decirlo con (Domínguez et al, 2015), debe ser flexible y adaptarse a las nuevas condicionantes que su actuación exige para la transferencia del conocimiento en las aulas

No se puede poner en duda la importancia de los estilos de aprendizaje en el proceso de enseñanza y aprendizaje. No obstante, no se puede ignorar la importancia que tiene el hecho de que los estudiantes enfrenten nuevos desafíos donde poder poner a prueba otros estilos de aprendizaje diferentes a los propios para que esto les ayude a enfrentar las materias de forma diferente (Gutiérrez,2018). 
Los estilos de aprendizaje se entienden como variables personales que, a mitad de camino entre la inteligencia y la personalidad, explican las diferentes formas de abordar, planificar y responder ante las demandas del aprendizaje (Camarero F, Buey F M, Herrero J. 2000, citado en Fernández, Beligoy, 2015).

\section{EL MODELO VAK}

Este constructo permite identificar el mejor de los tres canales de percepción: visual, auditivo, kinestésico. Con el avance del artículo y según las demás fuentes consultadas, se puede observar que, independientemente de los canales perceptivos utilizados, diferentes estudios arrojan tendencias hacia algún canal por encima de otro, la cantidad de información que el cerebro logra retener depende directamente de la metodología didáctica que el docente emplee, pues dependiendo de eso logrará estimular en mayor capacidad alguno de los tres canales perceptivos que se mencionan (Reyes, Molina,2017). Pero también del canal preferencial del estudiante porque si el chico no tiene claro su preferencia de aprendizaje puede costarle trabajo el decidir cómo aprende mejor la asignatura.

Visual: los sujetos que perciben desde este canal piensan en imágenes y tienen la capacidad de captar mucha información con velocidad, también son capaces de abstraer y planificar mejor que los siguientes estilos. Aprenden con la lectura y presentaciones con imágenes. $Y$ no se puede olvidar que en este siglo XXI las tecnologías privilegian esta forma de informar $y$ comunicar conocimientos o información.

- Auditivo: los sujetos que utilizan el canal auditivo en forma secuencial y ordenada aprenden mejor cuando reciben explicaciones orales y cuando pueden hablar y explicar determinada información a otra persona. Estos alumnos no pueden olvidar una palabra porque no saben cómo sigue la oración; además, no les permite relacionar conceptos abstractos con la misma facilidad que el visual. Este canal es fundamental en estudios de música e idiomas no así en ciencias duras o abstractas como la química.

- Kinestésico: son sujetos que aprenden a través de sensaciones y ejecutando el movimiento del cuerpo. Es el sistema más lento en comparación a los anteriores, pero su ventaja es que es más profundo, una vez que el cuerpo aprende determinada información le es muy difícil olvidarla; así, estos estudiantes necesitan más tiempo que los demás, lo que no significa un déficit de comprensión, sino solo que su forma de aprender es diferente (Flores, E. y Maureira, F., 2015 citado en Reyes, Molina 2017).

\section{LAS CARACTERÍSTICAS PRINCIPALES DEL MODELO HONEY -ALONSO ESTILOS ACTIVO, REFLEXIVO, PRAGMÁTICO}

Activo. Su aprendizaje es basado en la experiencia directa, por lo que se está siempre abierto a la experimentación, al trabajo en grupo, a los retos y se caracteriza por su implicación en la acción; quienes tienen preferencia por este estilo, se destacan por ser animadores, descubridores, improvisadores, creativos, líderes.

El estilo activo es característico de las personas que se implican plenamente y sin prejuicios en nuevas situaciones. Son de mente abierta, nada escépticos y se crecen ante los desafíos que suponen nuevas experiencias aburriéndose con las actividades a largo plazo. Son espontáneos, creativos innovadores, deseosos de aprender y resolver problemas.

Reflexivo. Estilo basado en la observación desde diversas perspectivas, considera todas las alternativas; son ponderados, concienzudos, analíticos, detallistas, previsores, cuestionadores, investigadores. 
El estilo reflexivo es característico de las personas que les gusta considerar las experiencias observadas desde diferentes perspectivas. Reúnen datos, analizándolos con detenimiento antes de llegar a una conclusión. Son prudentes y consideran todas las alternativas posibles antes de realizar un movimiento. Escuchan a los demás y no actúan hasta apropiarse de la situación cuando tienen que actuar sin planificar (González et al, 2018).

Teórico: Se basa en la conceptualización abstracta, en la formación de conclusiones e integra los hechos en teorías coherentes; quienes prefieren este estilo son metódicos, objetivos, críticos, estructurados, disciplinados, sistemáticos, sintéticos, perfeccionistas, y creadores de procedimientos.

En el estilo teórico, se tiende a ser perfeccionista, adaptando e integrando las observaciones dentro de teorías lógicas y complejas. Este colectivo es profundo en su sistema de pensamiento buscando la racionalidad y la objetividad. Buscan la racionalidad y la objetividad huyendo de los subjetivo y de lo ambiguo.

Pragmático: basado en la experimentación activa y búsqueda de aplicaciones prácticas; se caracteriza por el eclecticismo; quienes desarrollan este estilo son experimentadores, prácticos, directos, realistas, eficaces; disfrutan llevando a cabo lo aprendido, planificando acciones, organizando, situándose en el presente, solucionando problemas.

En el estilo pragmático se busca la aplicación práctica de ideas. Son personas que les gusta actuar rápidamente y con seguridad con aquellas ideas y proyectos que les atraen, aprovechando la primera oportunidad para experimentar (González et al, 2018).

La aplicación de los estilos de aprendizaje proporciona un perfil de los estudiantes que sirve para actuar (Escanero et al, 2019).
No se puede dejar de lado que la importancia de la teoría de los Estilos de Aprendizaje radica en su eficacia para el proceso de enseñanza y aprendizaje, como factor clave para el éxito académico de los estudiantes (Gutiérrez, 2018).

Los resultados encontrados por Castillo, plantean un reto importante para los docentes universitarios, ya que al ser los facilitadores del proceso enseñanza y aprendizaje es fundamental que tomen en cuenta las preferencias y características individuales de sus estudiantes, porque, al tener conocimiento de tales diferencias en el aula de clase, pueden desarrollar así un amplio abanico de estrategias pedagógicas que propicien la adquisición de conocimientos significativos en los estudiantes (Castillo, Mendoza, 2015).

La enseñanza centrada en el aprendizaje favorece que los/las docentes en el aula diseñen, incorporen y difundan diversas estrategias, acciones, técnicas y metodologías que lleven al estudiantado a asumir, construir, apropiarse y transformar la información para facilitar la comprensión de los diversos contenidos académicos (Sandoval, 2016).

Esta investigación tiene la finalidad de determinar los estilos de aprendizaje y los canales de percepción de los estudiantes del Programa Educativo de Químico Farmacéutico Biólogo.

\section{METODOLOGÍA}

A continuación se describe en forma sintética la metodología seguida para este estudio:

Unidad de Análisis: Facultad de Ciencias Químico Biológicas

Población de estudio: Estudiantes de la Facultad de Ciencias Químico Biologicas

Sujetos participantes: 92 estudiantes, con un rango de edad de 18 a 21 años, pertenecientes al año de ingreso 2017 del Programa Educativo de 
Químico Farmacéutico Biólogo de la Facultad de Ciencias Químico Biológicas de la Universidad Autónoma de Campeche.

\section{INSTRUMENTOS:}

1.- Cuestionario de estilos de aprendizaje del autor P. Honey y A. Mumford (CHAEA) (Alonso et al, 1994).

2.- Test de Sistema de Representación Favorito del Modelo de la Programación Neurolingüística de Bandler y Grinder. Este test Visual, Auditivo, Kinestésico (VAK) es para ayudar a descubrir la manera preferida de aprender. Cada persona tiene su manera preferida de aprender. Reconocer las preferencias ayudará a comprender las fuerzas en cualquier situación de aprendizaje (Robles, 2000).

\section{PROCEDIMIENTO}

Al inicio del ciclo escolar en el año 2017 a todos los estudiantes de nuevo ingreso al programa educativo de Químico Farmacéutico Biólogo se les aplicaron los dos cuestionarios, el de estilos de aprendizaje de CHAEA y el de Sistema de representación favorito, esta generación estuvo formada por tres grupos de primer semestre. Se les explico el motivo de la aplicación de estos instrumentos, antes de iniciar el llenado de cada uno de los cuestionarios, se les resolvió algunas dudas que surgieron en el momento de la aplicación. Terminando el llenado de los cuestionarios los estudiantes tuvieron su resultado de cada instrumento. Posteriormente estos resultados de los estudiantes se les informó a todos los docentes que les impartieron clases a todos los estudiantes que ingresaron en 2017.

\section{RESULTADOS}

Se presenta el análisis de resultados de la encuesta aplicada considerando la clasificación del estilo Honey - Alonso aplicados a los estudiantes de los tres grupos que ingresaron en el año 2017 a la licenciatura de Químico Farmacéutico Biólogo con la finalidad de obtener información, de la forma en que los estudiantes perciben los conocimientos en el aula.

Los estudiantes de los tres grupos que ingresaron en el año 2017 presentan los siguientes resultados:

\section{Estilo VAK 1" "A"ingreso 2017}

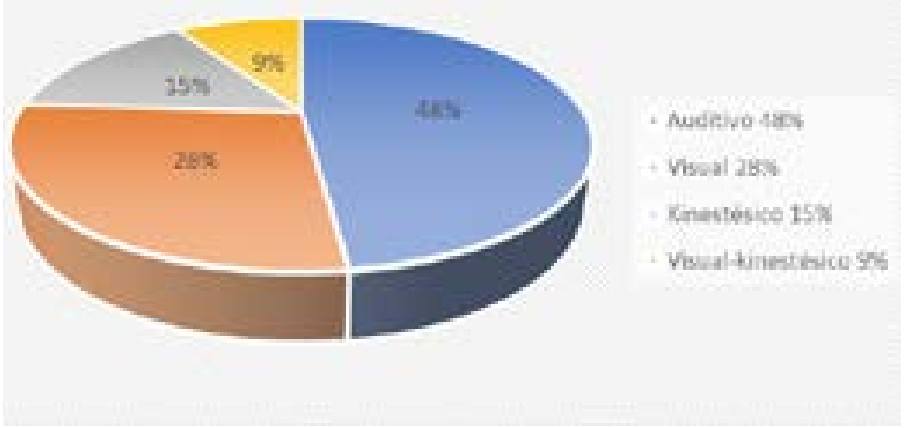

Figura 1 Estilo de aprendizaje VAK de los estudiantes del grupo " $A$ "que ingresaron en 2017

Como se aprecia en la figura 1 el estilo que predominó con $48 \%$ fue el canal de percepción auditivo este es cuando necesita escuchar su grabación mental paso a paso (Neira, 2015).

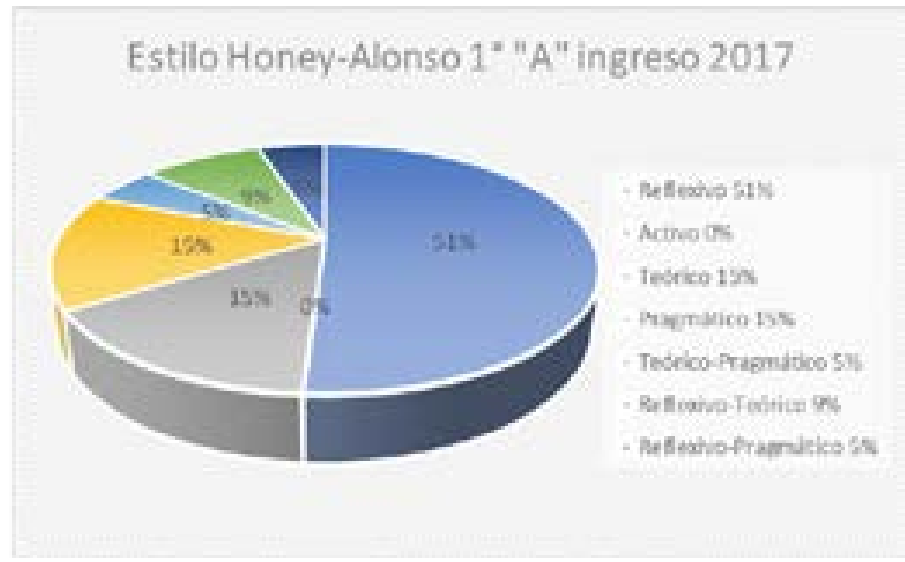

Figura 2 Estilo de aprendizaje Honey y Alonso de los estudiantes del grupo "A" que ingresaron en 2017 
La figura 2 muestra el porcentaje de los estudiantes de Químico Farmacéutico Biólogo respecto a su estilo de aprendizaje. El mayor porcentaje es $51 \%$ reflexivos y representa a los estudiantes reflexivos, quienes aprender mejor cuando pueden ofrecer observaciones y analizar la situación (ANUIES, 2006).

\section{Estilo VAK"B"ingreso 2017}
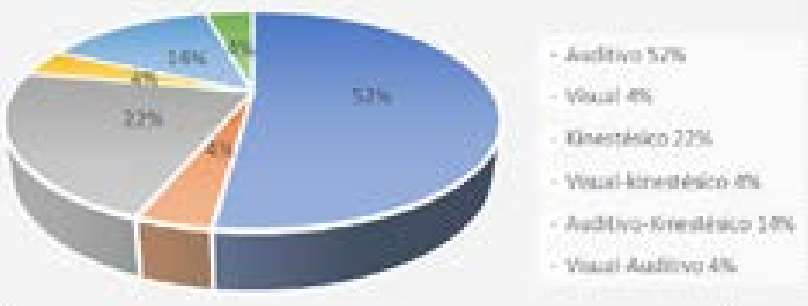

Vaud eufove ds

Figura 3 Estilo de aprendizaje VAK de los estudiantes del grupo "B" que ingresaron en 2017

Como se aprecia en la figura 3 el estilo que predominó con $52 \%$ fue el canal de percepción auditivo estos estudiantes aprenden mejor cuando reciben explicaciones orales $y$ cuando pueden hablar y explicar determinada información a otra persona (Flores, E. y Maureira, F., 2015 citado en Reyes, Molina, 2017).

\section{Estilo Honey-Alonso 1" B" ingreso 2017}
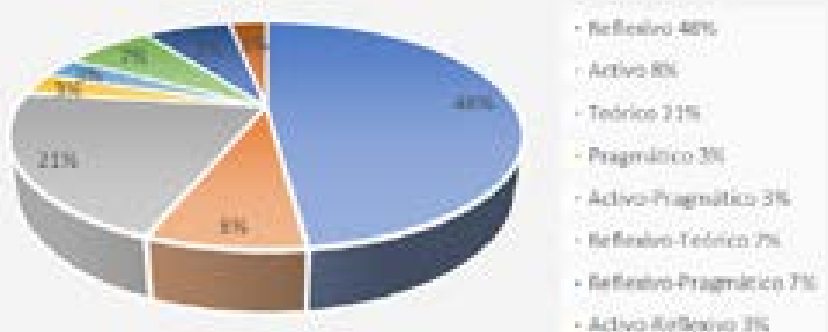

- Advoknowio as

Figura 4 Estilo de aprendizaje Honey y Alonso de los estudiantes del grupo "B" que ingresaron en 2017

La figura 4 muestra el porcentaje de los estudiantes de Químico Farmacéutico Biólogo respecto a su estilo de aprendizaje. El mayor porcentaje es $48 \%$ reflexivos y representa a los estudiantes reflexivos, característico de las personas que les gusta considerar las experiencias observadas desde diferentes perspectivas (González et al, 2018).

\section{Estilo VAK1"C" ingreso 2017}

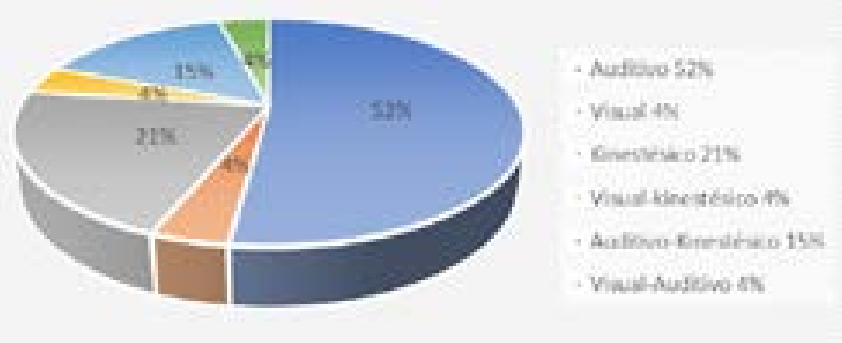

Figura 5 Estilo de aprendizaje VAK de los estudiantes del grupo " $C$ " que ingresaron en 2017

Como se aprecia en la figura 5 el estilo que predominó con $52 \%$ fue el canal de percepción auditivo, estos estudiantes no pueden olvidar una palabra porque no saben cómo sigue la oración; además, no permite relacionar conceptos abstractos con la misma facilidad que el visual (Flores, E. y Maureira, F., 2015 citado en Reyes, Molina, 2017).

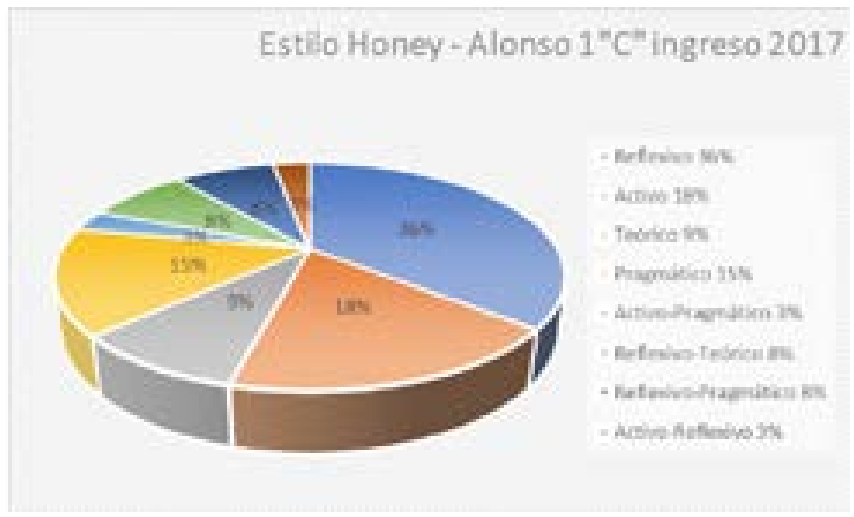

Figura 6 Estilo de aprendizaje Honey y Alonso de los estudiantes del grupo "C" que ingresaron en 2017 
La figura 6 muestra el porcentaje de los estudiantes de Químico Farmacéutico Biólogo respecto a su estilo de aprendizaje. El mayor porcentaje es $36 \%$ reflexivos y representa a los estudiantes reflexivos que son lo que reúnen datos, analizándolos con detenimiento antes de llegar a una conclusión. Son prudentes y consideran todas las alternativas posibles antes de realizar un movimiento (González et al, 2018).

Es importante destacar que según la literatura revisada los alumnos de CQB deberían ser visuales y reflexivos, sin embargo una de las cosas que sobresale en este trabajo es que son auditivos, y reflexivos, una posible explicación podría ser que las herramientas de trabajo de la enseñanza han ido cambiando, ya que las tecnologías de la comunicación cada vez impactan más auditivamente, esto no quiere decir que los jóvenes no usen el canal visual sino que su preferencia se orienta más a indagar y no a repetir cosas sin reflexionar, algo que era común en la forma de enseñanza, los docentes están utilizando otras estrategias que fomentan el uso de todos los canales de aprendizaje, también es importante mencionar que la parte activa o kinésis si bien no aparece en primer lugar, si está presente en todos los grupos estudiados y como se dijo anteriormente el conocimiento se fija a través del movimiento y la evocación auditiva o visual.

\section{CONCLUSIONES}

Considerando el estilo de aprendizaje Honey Alonso como referencia, se obtuvo que en los grupos A, B y C estudiados los estudiantes del Programa Educativo de Químico Farmacéutico Biólogo de la Universidad Autónoma de Campeche, tienen como estilo preferente de aprendizaje al "Reflexivo", caracterizándose por la pregunta a la que responden en el aprendizaje los estudiantes con un estilo predominante reflexivo es ‘¿por qué?' De ahí que las situaciones favorables para ellos son cuando pueden ofrecer observaciones y analizar la situación. Cuando pueden pensar antes de actuar. Desfavorables, cuando se les fuerza a ser el centro de atención. Cuando se les apresura de una actividad a otra, lo cual explica porque estos estudiantes pareciera que son tímidos o poco sociables sin embargo son jóvenes a los cuales no les gusta equivocarse por lo cual tardan en hablar y lo hacen solo después de un análisis previo a lo que se les pregunta.

Respecto al estilo de aprendizaje VAK, se encontró en los tres grupos estudiados que los estudiantes del Programa Educativo de Químico Farmacéutico Biólogo de la Universidad Autónoma de Campeche tienen como estilo preferente de aprendizaje el "Auditivo". Estos alumnos no pueden olvidar una palabra porque no saben cómo sigue la oración; además, no permite relacionar conceptos abstractos con la misma facilidad que el visual y podría pensarse que no tienen un aprendizaje significativo, lo cual en este trabajo parece quedar en entredicho.

Los estilos de aprendizaje proporcionan a los docentes información para tener un panorama amplio sobre sus estudiantes a los que enseñan $y$ les permite fortalecer estrategias para incrementar el aprovechamiento escolar en el nivel superior.

La información obtenida en este estudio se les proporcionó a los docentes de los tres grupos en estudio, para que consideraran estos aspectos en el diseño de estrategias de planeación docente, para que favorezcan su proceso de enseñanza y aprendizaje. Por lo que en estas estrategias se deben incluir actividades variadas que involucre los tres canales de aprendizaje: Auditivo, Visual, Kinestésico, así como los estilos Activo, Reflexivo, Teórico, Pragmático y fomentar así el uso de los estilos en los estudiantes en el proceso de enseñanza y aprendizaje en el aula, contribuyendo con esto a fortalecer el nivel de aprovechamiento en el Nivel Superior. 


\section{REFERENCIAS BIBLIOGRAFICAS}

Alonso, C., Gallego, D., Honey P. (1994). Los estilos de aprendizaje. Procedimientos de diagnóstico y Mejora. Ed. Mensajero. Bilbao, España

ANUIES. (2006). Antología del curso estilos de aprendizaje y estrategias de enseñanza. Universidad Autónoma de Campeche. México.

Castillo Díaz Marcio Alexander; Mendoza Aly, Jorge Luis. (2015). Estilos de aprendizaje en estudiantes universitarios: recursos informáticos como estrategia para su evaluación. UNAH INNOV@ No 42015 pág. 33-39 digital.

Domínguez Rodríguez, Heriberto de Jesús; Gutiérrez Limón, Jorge Alberto; Llontop Pisfil, Manuel; Villalobos Torres, David; Delva Exume, Jean Claude. (2015). Estilos de aprendizaje: un estudio diagnóstico en el centro universitario de ciencias económico-administrativas de la U de G. Revista de la Educación Superior. ANUIES. Volumen 44. Pp 121-140.

Escanero-Marcén, Jesús F., Soria, M. Soledad, Guerra-Sánchez, Manuel, \& Silva, Jackson. (2016). Comparación de los estilos de aprendizaje de los alumnos de medicina obtenidos con un nuevo cuestionario con los proporcionados por el cuestionario Honey-Alonso (CHAEA). FEM: Revista de la Fundación Educación Médica, 19(1), 19-26. Recuperado en 14 de octubre de 2019, de http://scielo.isciii.es/scielo. php? script=sci_arttext\&pid=S2014 $98322016000100006 \&$ lng=es\&tlng=es.

Fernández, Víctor, \& Beligoy, Mariela. (2015). Estilos de aprendizaje y su relación con la necesidad de reestructuración de las estrategias de aprendizaje de los estudiantes universitarios de primer año. FEM: Revista de la Fundación Educación Médica, 18(5), 361366. https://dx.doi.org/10.4321/S20149832201500060001169820110005000 $13 \&$ Ing=es\&tlng=es.

Gutiérrez Tapias, Mariano, (2018). Estilos de aprendizaje, estrategias para enseñar. Su relación con el desarrollo emocional y "aprender a aprender". TENDENCIAS PEDAGÓGICAS N³1 2018. España. P .94.

González Garza, Beatriz, Hernández Castañón, Ma. Alejandra, \& Castrejón Reyes, Victorina. (2018). Estilos de aprendizaje para el desarrollo de competencias en estudiantes de la Licenciatura en Enfermería. RIDE. Revista Iberoamericana para la Investigación y el Desarrollo Educativo, 8(16), 351369. https://dx.doi.org/10.23913/ride. v8i16.345

Neira Silva J. (2015). Visual, Auditivo o kinestésico los alumnos. Orientación Andújar. Perú.

Robles, Ana. (2000). Estilos de aprendizaje: como seleccionamos y representamos la información. Disponible en http:// www.galeon.com/aprenderaaprender/ general/indice.html.(consultado en 2012). México.

Reyes, L., Céspedes, G., Molina, J. (2017). Tipos de aprendizaje y tendencia según modelo VAK. TIA, 5(2), Bogotá -Colombia. pp. 237-242.

Sandoval Palomares Jessica. Los estilos de aprendizaje, una revisión desde la neuropsicología. Ciencia y Tecnología Universitaria. Año 4 Número 1 septiembre - diciembre 2016.p1. México. 